\title{
Automated Weighing System for the Health Care of Guinea Pigs (Cavia Porcellus)
}

\section{Sistema de Pesaje Automatizado para el Cuidado del Estado de Salud en Cuyes (Cavia Porcellus)}

I International Seminar of Livestock and Agroindustrial Production ESPOCH 2020

Corresponding Author:

J. Barco-Jiménez

jebarco@unicesmag.edu.co

Published: 2 September 2021

Production and Hosting by

Knowledge E

(c) J. Barco-Jiménez et al. This article is distributed under the terms of the Creative Commons Attribution License, which permits unrestricted use and redistribution provided that the original author and source are credited.
S OPEN ACCESS
J. Barco-Jiménez ${ }^{1}$, M. Martínez $z^{1}$, and A. L. Solarte ${ }^{2}$

${ }^{1}$ Facultad de Ingeniería, Universidad CESMAG, Pasto, Colombia

${ }^{2}$ Sistema Gestión de Calidad, Universidad CESMAG, Pasto, Colombia

\section{Abstract}

It is estimated that in Nariño (Colombia) there are 20,000 small guinea pig farms, which provide livelihoods for thousands of families and generate monthly transactions of a thousand million Colombian pesos. This sector has a trend of constant growth. However, the production of guinea pigs (Cavia porcellus) is done by hand due to the lack of technification of this sector. A problem identified in the breeding of these animals is weight loss, which can be caused by injuries in the traditional weighing process. In this process, the animal is immobilized and manually taken to the scale, which can cause injuries, stress, weight variations, reduced mobility and, in the worst cases, death. Being aware that these problems could be solved through technology, this article presents the design of an automated weighing and identification system that allows producers to carry out the weighing process without human intervention, with the purpose of safeguarding the animal's health. The article also discusses the parameter design and information about guinea pigs, from a structural and operative point of view. Some experimental results are shown which gave rise to a patent. Finally, conclusions about the reach and use of the device are presented.

Keywords: automated weighing system, RFID tagging, guinea pigs, Cavia porcellus.

\section{Resumen}

Se estima que en Nariño (Colombia) existen 20.000 criaderos pequeños de cuyes que son la base económica de miles de familias los cuales producen transacciones mensuales por mil millones de pesos colombianos. Un sector que está en constante crecimiento. Sin embargo, la producción de cuyes (Cavia porcellus) se realiza de forma artesanal debido a la falta de tecnificación. Una problemática identificada en la cría de estos animales es la pérdida de peso que puede ocasionarse por lesiones causadas en el pesaje de forma tradicional. En este proceso, el animal es inmovilizando y llevado manualmente hacia la báscula, lo que puede ocasionar lesiones, reducción de movilidad y hasta la muerte. Conscientes que dichas problemáticas podrían solucionarse a través de la tecnología, en este artículo se plantea el diseño de un sistema de pesaje e identificación automatizado que permita a los productores realizar el proceso de pesaje sin intervención humana, con el propósito de mantener el estado de salud del animal. Para esto se presenta la información recopilada y parámetros establecidos para realizar el diseño, desde un punto de vista estructural y operativo. Además, se presentan algunos resultados experimentales que dieron origen a una Patente del dispositivo, y finalmente algunas conclusiones sobre el alcance y uso del dispositivo propuesto.

Palabras Clave: sistema de pesaje automatizado, identificación RFID, cuyes de granja, Cavia porcellus. 


\section{Introducción}

El cuy (Cavia Porcellus) es originario de Suramérica estableciéndose principalmente en diferentes zonas como Bolivia, Colombia, Ecuador, Perú. Desde hace por lo menos 3000 años, fue utilizado como una importante fuente de alimentación para los pueblos aborígenes, y posteriormente fueron domesticados [1]. En la actualidad el cuy se cría en zonas rurales y suburbanas de estos países. Su periodo de supervivencia es aproximadamente de 8 años, sin embargo, alcanzan su madurez en peso en pocos meses (6 meses) [2]. Cuando se habla de este animal no se puede especificar simplemente una raza, debido a la diversidad de cruces que han tenido estos animales desde hace muchos años de manera incontrolada. Por lo tanto, se describen los tipos de animales que se han identificado en la literatura [3]. De acuerdo al pelaje y conformación del cuerpo hay seis tipos [4-6], sin embargo se describen solamente los animales objeto de estudio, por el límite de extensión. Tipo 1: De pelo corto, lacio y pegado al cuerpo pudiendo presentar un remolino en la frente. Este es uno de los tipos que presentan mejores características para producción de carne. Sus incrementos de peso son superiores a los de los tipos 3 y 4 . De acuerdo a la conformación del cuerpo hay dos tipos: Tipo A: Forma redondeada, cabeza corta y ancha, temperamento tranquilo. Son animales para la producción de carne que al cabo de tres meses alcanzan un peso ideal para el sacrificio. Por otro lado, se debe describir la raza correspondiente de los animales objeto de estudio. Raza Perú: Es una raza pesada, con desarrollo muscular marcado, es precoz y eficiente convertidor de alimento. El color de su capa es alazán con blanco; puede ser combinada o fajada, por su pelo liso corresponde al Tipo A. Puede o no tener remolino en la cabeza, orejas caídas, ojos negros, y, dentro de este tipo, puede haber también cuyes de ojos rojos, lo que no es recomendable. Estos presentan las siguientes características físicas y biológicas que son pertinentes para este estudio: peso (max.) de $1500 \mathrm{~g}$, ancho (max.) de $10 \mathrm{~cm}$, largo (max.) de $25 \mathrm{~cm}$, alto (max.) de 10 $\mathrm{cm}$.

En el departamento de Nariño, la producción de cuyes se realiza de forma artesanal debido a la falta de automatización y tecnificación en las granjas productoras [7], lo cual genera pérdida de competitividad en comparación con los procesos de producción de medianas especies a nivel nacional, que están optimizados y apoyados tecnológicamente para producir grandes volúmenes a un bajo precio. Estas barreras técnicas generan dificultad para manejar la producción del cuy en grandes cantidades y obtener un beneficio de reducción de costos al aumentar la producción [8, 9]. Específicamente, una problemática, que manifiestan los productores locales de cuyes de Nariño, es la pérdida de peso y disminución de salud del animal debido a lesiones causadas en el proceso de pesaje de forma tradicional. En este proceso el animal es acorralado e inmovilizando con una bolsa de tela y llevado manualmente hacia la báscula, a veces, produciéndole lesiones (menores o mayores) que conllevan a enfermedades que reducen su salud. Por ejemplo: Pueden perder movilidad por lesiones en sus extremidades y/o columna dificultando el acceso a la comida, tienen mayor probabilidad de contagio de enfermedades dado que se usa una misma bolsa para atrapar a todos los animales, se genera mayor estrés físico en el proceso, y en el peor de los casos cuando el proceso se sale de control, se puede ocasionar la muerte del 
animal. Así, se debe procurar que en la producción del cuy, mayor cuidado y buscar el acondicionamiento de los galpones para mejorar el control de factores internos y externos [10].

Actualmente, los productores no han buscado otros métodos para realizar el proceso de pesaje debido a la escasez de recursos. Por otro lado, no se han desarrollado dispositivos relacionados con la tecnificación del pesaje de animales de tamaño pequeño, por el contrario, existen varias alternativas comerciales para el pesaje de animales de tamaño mediano y grande [11-13], y otros aún en desarrollo que pueden ser referentes para buscar una solución a la problemática mencionada [14-18]. En general, la tecnificación sobre el proceso de producción de cuyes se ha enfocado en mantener condiciones favorables para el crecimiento por medio del control de temperatura, ventilación e iluminación. No obstante, muchos referentes mencionan la importancia de mantener al cuy en óptimas condiciones físicas, sin lesiones o marcas, para que no pierda su valor comercial. Por otro lado, se expone la importancia de llevar un registro continuo de peso para identificar las diferentes fases en las que se encuentra el animal, y tomar decisiones sobre diferentes aspectos, tales como: la venta del animal (fase de maduración completa), o cambio de lote, cambio de alimentación, entre otros [19].

En mérito de lo expuesto, este proyecto tiene el propósito de brindar una herramienta tecnológica de pesaje y monitoreo automatizado que facilite la producción en masa y ofrezca condiciones para reducir el costo de producción, haciendo el precio más competitivo en un contexto nacional con respecto a productos equivalentes.

Este artículo se divide de la siguiente manera: En primera instancia se presenta el lugar y el tipo de animal que fue objeto de estudio. Después se presentan los requerimientos y el diseño general del dispositivo de identificación y pesaje de cuyes, desde los puntos de vista estructural y operativo. Finalmente, se muestran resultados experimentales realizados en una finca de producción de cuyes ubicada en San Fernando - Nariño.

\section{Generalidades y Razas}

Esta investigación se desarrolló en la granja experimental del Corregimiento de San Fernando en el Municipio de San Juan de Pasto, Colombia, ubicado en las coordenadas: ${ }^{\circ} 12^{\prime} 12.6$ ” $\mathrm{N} 77^{\circ} 13^{\prime} 33.7$ 'W, con una altura de 2800 m.s.n.m., con una temperatura promedio de $14^{\circ} \mathrm{C}$. A continuación, se presenta las generalidades y razas de cuyes que se tuvieron en cuenta en este estudio.

\subsection{Diseño del Sistema Automatizado de Identificación y Pesaje para Cuyes (SAIPC)}

Para el diseño del sistema de identificación y pesaje de cuyes se tuvieron en cuenta, principalmente, las características físicas de los cuyes que se producen en la región (raza, tipo, tamaño, entre otros), y las características de las granjas nariñenses (tamaño galpón, temperatura, cantidades, entre otros). Estas características se convierten en 
los parámetros de diseño que permitirán desarrollar un dispositivo que se ajuste a las necesidades del sector de producción local de cuyes. No obstante, estos parámetros pueden ser ajustados para otras razas y tipos de cuyes, cumpliendo el mismo objetivo: La automatización del pesaje sin lastimar al animal.

\subsubsection{Diagrama de bloques funcional del SAIPC}

El diagrama de la Figura 1 presenta un diseño intuitivo que responde a las características de los cuyes, las condiciones de las granjas nariñenses, y responde a la manera en que los productores locales realizan el proceso de pesaje. El SAIPC se compone de tres partes principales: (1) componentes de pesaje, (2) componentes de identificación y (3) el sistema de procesamiento que permite integrar la interfaz de usuario y la tarjeta de almacenamiento de datos.
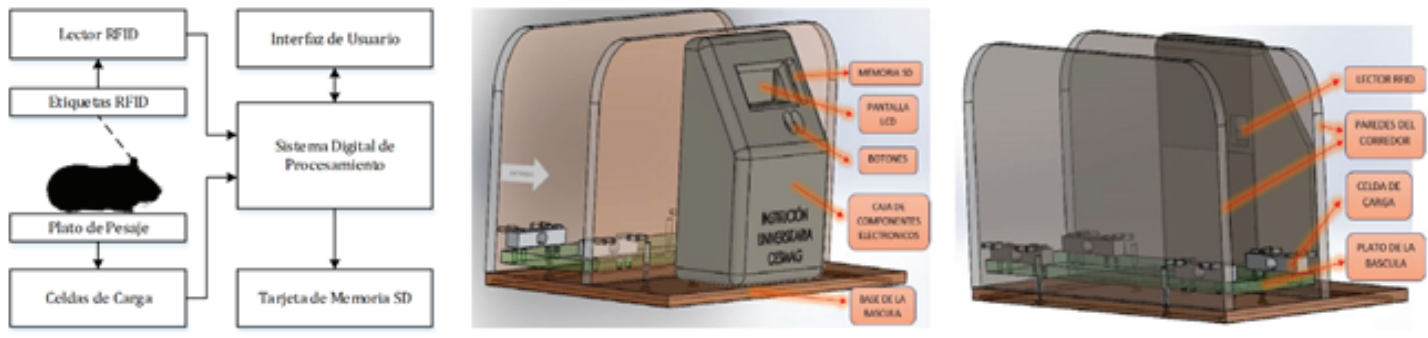

Figure 1

Diagrama de bloques del sistema de identificación y pesaje de cuyes.

1. Componentes de pesaje: Se diseña para que la medida de peso se pueda capturar de forma precisa y rápida. En este caso se utilizan celdas de carga ya que permiten obtener una medición casi instantánea (en el orden de los milisegundos), confiable y con bajo error de medición. La celda de carga traduce la fuerza ejercida por el peso del animal en una señal electrónica que es interpretada por el circuito electrónico de pesaje y convertida a gramos para que pueda ser visualizada. En total el sistema tiene 4 celdas de carga ubicadas en cada una de las esquinas sosteniendo el mismo.

2. Componentes de identificación: Para implementar la identificación se utiliza tecnología RFID (Radio Frequency IDentification), muy utilizando en la producción de animales para realizar el seguimiento y trazabilidad de animales. El uso de la tecnología RFID requiere de dos componentes, una etiqueta RFID que el animal debe usar constantemente, y un lector que permita leer el número de dicha etiqueta (Figura 1). En este caso, se utiliza etiquetas RFID similares a un botón (radio de 0,7 $\mathrm{cm}$ ) que puede ser adherido al animal como un arete. Estas contienen antenas para permitirles recibir y responder a peticiones por radiofrecuencia desde un lector RFID. No requieren alimentación eléctrica interna (etiquetas RFID pasivas) y contienen un código o número identificador único que permite diferenciar un animal de otro. El lector del RFID del sistema de identificación pesaje para cuyes transmite y recibe una respuesta con el número de la etiqueta RFID del animal. 
Particularmente, en este dispositivo se utiliza el lector RFID ID-12A que opera a una frecuencia de $125 \mathrm{kHz}$, y con cobertura alrededor de los $10 \mathrm{~cm}$.

3. Sistema digital de procesamiento, interfaz y memoria: Se encarga de recibir e interpretar las señales eléctricas provenientes de los componentes de pesaje e identificación para enviarlas a la pantalla LCD de la interfaz de usuario y visualizar los datos peso y número de identificación del animal. Adicionalmente, gestiona el guardado de los datos en la memoria SD. El sistema digital de procesamiento está basado en la plataforma de desarrollo ARDUINO MEGA 2560 rev 2.0 [20].

\subsubsection{Diagrama estructural del SAIPC}

A continuación, se describen los componentes estructurales más importantes del sistema SAIPC.

1. Plato y corredor de pesaje: El plato de pesaje debe ser lo suficientemente grande para contener completamente a un cuy (en etapa de madurez) parado en sus cuatro extremidades. El plato se ubica por debajo de los sensores de peso (celdas de carga) suspendido de los mismos y tiene la función de sostener al animal mientras su peso es registrado por el circuito electrónico. Tomando como referencia a los cuyes del Tipo 1 y A se definen las siguientes dimensiones del plato de pesaje: $39 \times 9 \times 1 \mathrm{~cm}$, y del corredor de pesaje de $40 \times 1 \times 20 \mathrm{~cm}$.

2. Base y caja de componentes electrónicos: La base permite sostener todos los elementos del sistema. Esta brinda el soporte al corredor y plato de pesaje manteniéndolos a la menor distancia posible del suelo ( $5 \mathrm{~cm}$ aproximadamente), para facilitar el ingreso de los cuyes dadas su característica de altura de $10 \mathrm{~cm}$ promedio. La caja de componentes electrónicos permite mantener el sistema digital de procesamiento e interfaz de usuario en condiciones funcionales, protegiendo de la suciedad, humedad, caída de líquidos que pueden ocurrir en un galpón. Además, evita que los animales puedan alcanzar y dañar los componentes electrónicos.

\subsection{Funcionamiento del Sistema Automatizado de Identificación y Pesaje para Cuyes (SAIPC)}

Primero, se debe ubicar estratégicamente el dispositivo de pesaje en un sector del galpón por donde el animal pueda desplazarse para recibir su alimento, o en cualquier ubicación que suponga el desplazamiento del animal por encima del plato de la báscula. Cuando el animal se acerque a la báscula, el pasillo debe dirigirlo hacia el plato de pesaje de la báscula, posteriormente, cuando el animal llegue hasta la posición central del plato del báscula, el lector RFID debe realizar la detección de la etiqueta RFID del animal y el circuito electrónico debe registrar el número de identificación y realizar la lectura de peso por medio de las celdas de carga, finalmente, los datos de identificación y peso del animal son visualizados en la pantalla LCD y guardados en la memoria SD junto con los datos de hora y fecha en que ocurrió el proceso. 


\section{Resultados y Discusión}

Tras una primera experimentación con el registro del SAIPC en un galpón de cuyes, se realizaron dos modificaciones sobre la estructura para mejorar el funcionamiento del sistema. Específicamente, se ubicaron escalones a la entrada y la salida del corredor de pesaje para facilitar el acceso y salida del animal del sistema de pesaje. Igualmente, se puede observar dos estructuras curvas pegadas en el corredor de pesaje. Estos elementos actúan como bretes haciendo que el animal se desplace lentamente sobre el corredor de pesaje, mejorando el registro de peso del animal, de igual, forma los bretes dirigen la cabeza del animal hacia el detector RFID, mejorando la detección del mismo.

Se realizaron pruebas en un entorno real, comparando las medidas obtenidas en con una báscula de pesaje tradicional y el SAIPC. En primer lugar se realizó el registro manual de peso para los animales del galpón (tomando 20 como muestra). Posteriormente se instalaron etiquetas RFID a los mismos animales y se dejaron en el galpón junto con el sistema de pesaje automatizado. Después de realizar varias pruebas de pesaje, en la siguiente tabla se muestran los resultados promedio de 3 mediciones para cada animal.

\section{Table 1}

Mediciones de peso en cuyes.

\begin{tabular}{|l|l|l|l|l|}
\hline & $\begin{array}{l}\text { Peso [g] báscula } \\
\text { tradicional }\end{array}$ & Peso [g] SAIPC & Error absoluto [g] & Error porcentual [\%] \\
\hline $\mathbf{1}$ & 1197 & 1256,7 & 60 & $5 \%$ \\
\hline $\mathbf{2}$ & 1574 & 1521,5 & 53 & $3 \%$ \\
\hline $\mathbf{3}$ & 1248 & 1221,9 & 26 & $2 \%$ \\
$\mathbf{4}$ & 1488 & 1505,3 & 17 & $1 \%$ \\
\hline $\mathbf{5}$ & 1572 & 1450,4 & 122 & $8 \%$ \\
\hline $\mathbf{6}$ & 1615 & 1626,6 & 12 & $1 \%$ \\
\hline $\mathbf{7}$ & 1628 & 1628,3 & 0 & $0 \%$ \\
\hline $\mathbf{8}$ & 1776 & 1601,3 & 175 & $10 \%$ \\
\hline $\mathbf{9}$ & 1058 & 1231,0 & 173 & $16 \%$ \\
\hline $\mathbf{1 0}$ & 1194 & 1278,2 & 84 & $7 \%$ \\
\hline $\mathbf{1 1}$ & 1542 & 1590,9 & 49 & $3 \%$ \\
\hline $\mathbf{1 2}$ & 1489 & 1596,6 & 108 & $7 \%$ \\
\hline $\mathbf{1 3}$ & 1475 & 1593,1 & 118 & $8 \%$ \\
\hline $\mathbf{1 4}$ & 1387 & 1515,6 & 129 & $9 \%$ \\
\hline $\mathbf{1 5}$ & 1565 & 1549,2 & 16 & $1 \%$ \\
\hline $\mathbf{1 6}$ & 1357 & 1475,4 & 118 & $9 \%$ \\
\hline $\mathbf{1 7}$ & 1879 & 1969,1 & 90 & $5 \%$ \\
\hline $\mathbf{1 8}$ & 1696 & 1848,7 & 153 & $9 \%$ \\
\hline $\mathbf{1 9}$ & 1489 & 1637,5 & 149 & $10 \%$ \\
\hline $\mathbf{2 0}$ & 1282 & 1385,5 & 104 & $8 \%$ \\
\hline & & & & \\
\hline
\end{tabular}


De la Tabla 1, se calcula la media del error (MAE) del dispositivo en de $\pm 87,7 \mathrm{~g}$, en términos de porcentaje es de $6,0 \%$. Los valores de error mencionados son aceptables en comparación con los sistemas de medición de peso tradicionales que se encuentran en el comercio local, lo cuales tienen errores de medición en el orden de los $\pm 100 \mathrm{~g}$. Por otro lado, se debe mencionar el parámetro de conservación de estado de salud del animal. Al realizar el experimento con el sistema de pesaje automatizado se notó que el animal no sufre ningún daño físico. Adicionalmente, es importante mencionar que los animales no hacen uso del SAIPC inmediatamente. Estos tienen que ser estimulados con alimento para que se desplacen por encima del plato de pesaje. Tras obtener validar el funcionamiento en campo del dispositivo, se procedió a solicitar la patente ante la entidad de protección de propiedad intelectual de Colombia (Superintendencia de Industria y Comercio), la cual fue otorgada en julio de 2020.

\section{Conclusiones}

El sistema automatizado de identificación y pesaje para cuyes presenta resultados satisfactorios en el registro del peso del animal e identificación de la etiqueta RFID, con un error de medición por debajo de los $\pm 100 \mathrm{~g}$, el cual es un error de medición típico de los dispositivos de medición encontrados en el comercio local. Tras el desarrollo de varios experimentos de medición se puede observar que el SAIPC permite realizar el pesaje de los animales sin necesidad de la intervención del productor, evitando que el animal pueda lesionarse por manipulación inadecuada o accidental, conservando el estado de salud del animal.

\section{Agradecimientos}

Por parte de los autores se expresa un agradecimiento a la UNIVERSIDAD CESMAG (Pasto - Nariño - Colombia) por la financiación del proyecto 'Desarrollo de un Sistema Automatizado de Identificación y Pesaje para Cuyes'.

\section{References}

[1] Quijandria B, Muscari J, Robison OW. Selection in guinea pigs: III. Correlated responses to selection for litter size and body weight. Journal of Animal Science. 1983;56(4):829-832.

[2] Rodríguez H, Gutiérrez G, Palomino M, Hidalgo V. Características maternales al nacimiento y destete en cuyes de la costa central del Perú. Revista de Investigaciones Veterinarias Del Perú. 2015;26(1):77-85.

[3] Yamada G, Bazán V, Fuentes N. Parámetros productivos de cuyes G en la costa central del Perú. Revista de Investigaciones Veterinarias Del Perú. 2018;29(3):877-881.

[4] Castro HP. Sistemas de crianza de cuyes a nivel familiar-comercial en el sector rural. Utah: Benson Agriculture and Food Institute; 2002.

[5] Quispe AS. Manejo técnico de la crianza de cuyes en la sierra del Perú. Arequipa; 2015.

[6] de Zaldívar, CL. Crianza de cuyes. Lima; 1994.

[7] Holandeses asesoran a Pasto en la cría de cuyes. Redacción Periódico El Tiempo. 2015 February 9. Available from: https://www.eltiempo.com/archivo/documento/CMS-15222239.

[8] Burgos W, Solarte C, Cerón M. Efecto del tamaño de camada y número de parto en el crecimiento de cuyes (Cavia porcell us Rodentia: caviidae). Revista Lasallista de Investigación. 2010;7(2):47-55.

[9] Elijah O, Rahman TA, Orikumhi I, Leow CY, Hindia MN. An overview of Internet of Things (loT) and Data Analytics in Agriculture: Benefits and challenges. IEEE Internet of Things Journal. 2018;5(5):3758-3773. 
[10] Zambrano O. Costos de producción de crianza artesanal y tecnológica del cuy (Cavia porcellus) en Cajamarca [Tesis de Maestría]. 2015.

[11] PCE Ibérica S.L. Instrumentación. Balanza para animales PCE PS 75XL. 2018.

[12] PCE Ibérica S.L. Instrumentación. Balanza PCB 6000-0. 2018.

[13] PCE Ibérica S.L. Instrumentación. Balanza PCE-PS 150XL. 2018.

[14] Liu T, Teng G, Fu W. Research and development of pig weight estimation system based on image, 2011. International Conference on Electronics, Communications and Control, ICECC 2011 - Proceedings. IEEE. 2011.

[15] Vaughan J, Green PM, Salter M, Grieve B, Ozanyan KB. 2017. Floor sensors of animal weight and gait for precision livestock farming. Proceedings of IEEE Sensors; 2017 December 1-3.

[16] Farooq MS, Riaz S, Abid A, Abid K, Naeem MA. 2019. A Survey on the Role of loT in Agriculture for the Implementation of Smart Farming. IEEE Access, 7, 156237-156271.

[17] Liu T, Teng G, Fu W. Research and development of pig weight estimation system based on image. 2011 International Conference on Electronics, Communications and Control, ICECC 2011 - Proceedings. p. 2774-2777.

[18] Harper BD. Digital farming: What does it really mean? CEMA - Europen Agricultural Machinery; 2017. pp. 1-9.

[19] Oshiro HR. Tecnificación de la crianza de cuyes para el mercado Nacional. Lima; 2008.

[20] Guadalupi A. Schematics Ardun. 2019. Available from: https://content.arduino.cc/assets/MEGA2560_ Rev3e_sch.pdf 